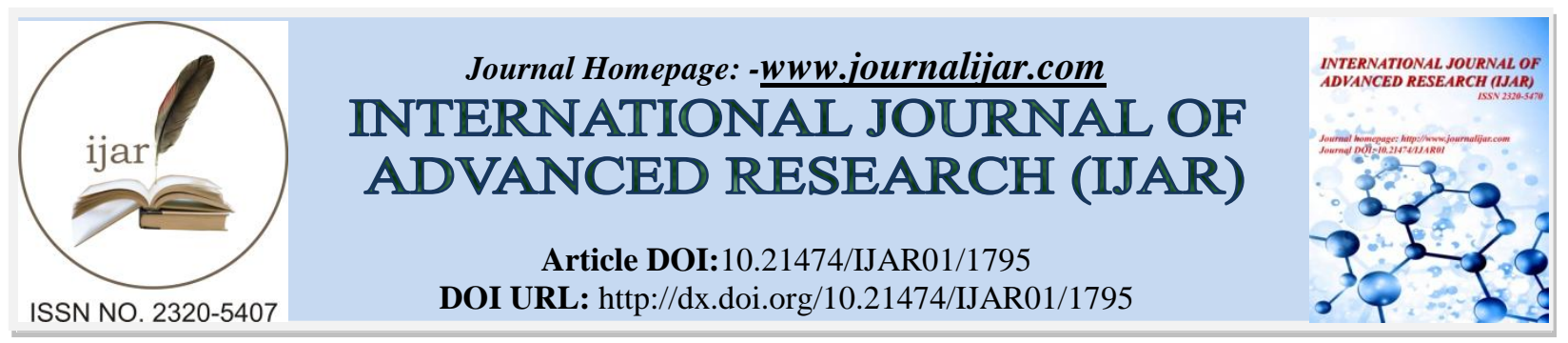

RESEARCH ARTICLE

\title{
EXTENSIVE LEAKAGE FROM A TYPE B AORTIC DISSECTION WITH MASSIVE LEFT HEMOTHORAX.
}

\author{
Yasser El-Ghoneimy ${ }^{1}$, Abdullah Al-Otaibi ${ }^{2}$, Mohannad Al-Qahtani ${ }^{2}$ and Haider Al-Herz ${ }^{2}$. \\ 1. Associate Professor and Cardiothoracic Surgeon, University of Dammam, King Fahd Hospital of the \\ University, Al-Khobar P.O. Box 2208, Saudi Arabia. \\ 2. Medical intern, University of Dammam, King Fahd Hospital of the University, Al-Khobar, Saudi Arabia.
}

\section{Manuscript Info \\ Manuscript History \\ Received: 12 August 2016 \\ Final Accepted: 22 September 2016 \\ Published: October 2016}

Key words:-

\section{Abstract}

Aortic dissection, or a teat of the aorta, results in the efflux of blood into the mediastinum and, in some instances, results in a hemothorax. A 62-year-old man was referred to the department of cardiothoracic surgery for exploration of left pleural opacity. Computed tomography angiography (CTA) revealed a type B aortic dissection according to the Stanford classification. Emergency trans-endovascular aortic repair was performed by implanting two large stents and a left chest tube to drain the massive hemothorax, which drained $1800 \mathrm{~mL}$ of fluid. Aortic dissection can result in a hemothorax, particularly in high-risk patients, such as elderly patients and those with systemic hyper tension. As demonstrated in our case, CTA reformatting is crucial for surgical planning and provides information for follow-up analysis and measurements.

Copy Right, IJAR, 2016,. All rights reserved.

\section{Introduction:-}

Acute dissection of the thoracic aorta, one of the most common causes of aortic emergencies, requires immediate diagnosis and treatment (Castaner et al. 2003). There are two generally known classification systems for dissections: the Stanford and DeBakey systems. The Stanford system is more widely used and classifieds is sections that involve the ascending aorta as type A, regardless of the site of the primary intimal tear, and all other dissections as type B (Dake et al., 2013). The most important predisposing factor for acute aortic dissection is systemic hyper tension (Larson et al., 1984).Pain, usually in the chest or back, is the most common manifestation and occursinover90\% of patients (Pape et al., 2015). Chest pain was significantly more common in patients with type A (Pape et al., 2015), while both back pain and abdominal pain were significantly more common in patients with type B dissections (Hagan et al., 2000). Routine blood tests often do not provide a basis for diagnosis and imaging studies are not performed until the patient is stabilized medically. Clinicians often perform transesophageal echocardiography (TEE) in the emergency room for patients who present with acute chest pain and/or are thermodynamically unstable (Cigarroa et al., 1993. In cases of aortic dissection, hemothorax secondary to rupture is rare (Langin et al., 1994). Here, we present a case with ruptured Stanford type B aortic dissection that resulted in massive left hemothorax.

\section{Case History:-}

A 62-year-old British engineer who presented with sudden onset of chest and back pain associated with nausea and vomiting early in the morning $(6: 00 \mathrm{am})$. The patient was referred to a private hospital where he received analgesics 
and was found to have severe hypertension that was unresponsive to medications. CT of the chest was performed (Figure 1), which exhibited extensive dissection of the thoracic aorta starting at the distal end of the aortic arch at the origin of the left sub clavian artery. Echocardiography showed left ventricular (LV) hypertrophy with good LV function and no valvular heart disease. The patient was referred to another hospital after a few hours because there was no facility that could manage this case at the private hospital. Unfortunately, no additional care was provided to the patient.

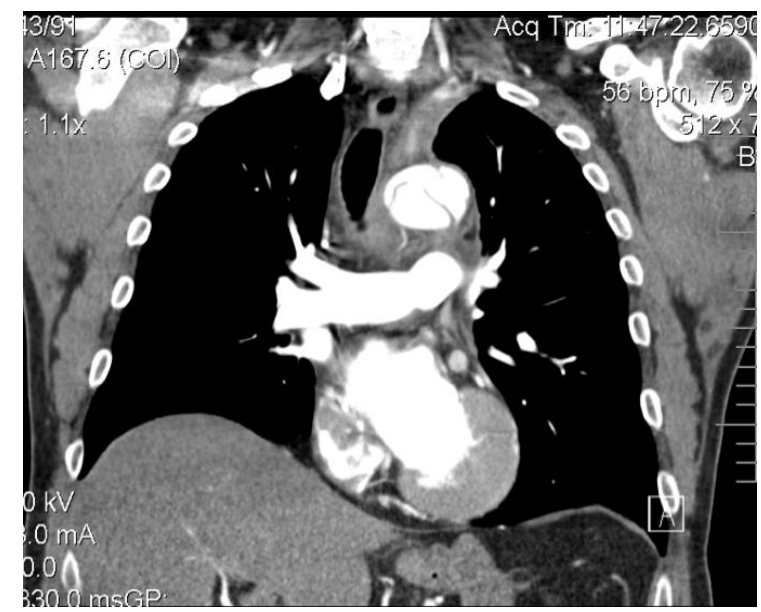

Figure 1:- CT Angiography (coronial view) showing the beginning of a dissection at the distal aortic archwith true and false lumen.

The patient was referred to our hospital late in the evening 14 hours later. Upon arrival to the ER, the patient collapsed with severe hypotension, confusion, severe respiratory distress, and symptoms of hypoperfusion. The patient was immediately resuscitated, intubated and placed on mechanical ventilation. Chest X-ray and CT angiography (Figure 2) were repeated, which indicated the presence of an extensive type B aortic dissection with a large false lumen and a narrow true lumen and no perfusion to the left kidney. Furthermore, there was marked diminished air entry to the left lung because of the presence of a large hemothorax. Renal functions deteriorated, as indicated by elevated BUN and serum creatinine levels.

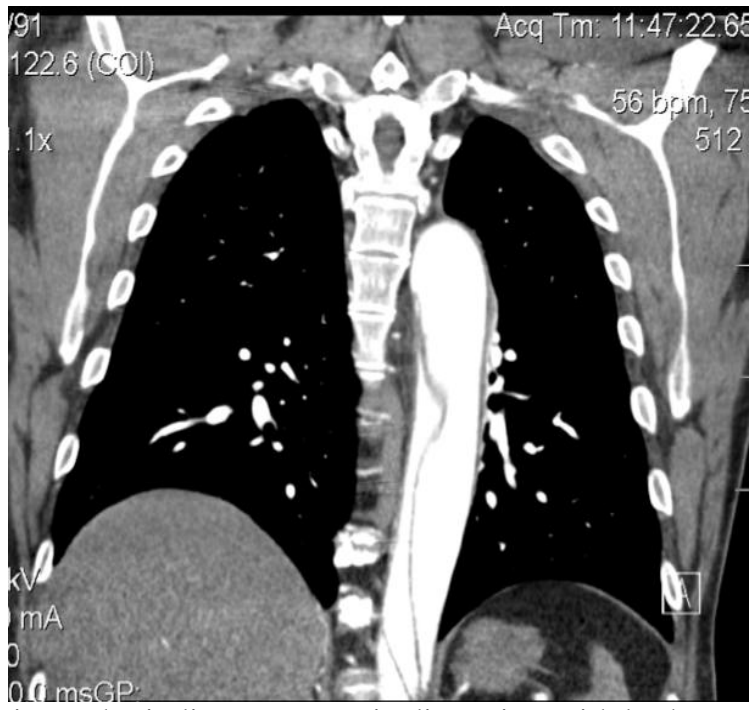

Figure 2:- CT angiography indicates an aortic dissection with both true and false lumens.

The patient required inotropic support and underwent immediate surgery. Under general anesthesia, transendovascular aortic repair(TEVAS) was performed using a right femoral approach. Furthermore, two large stents were placed in the descending thoracic aorta at the left sub clavian artery and in the upper abdominal aorta to ensure patency of the true lumen and to maintain flow to abdominal aorta branches (Figure 3). A left side chest tube was 
also inserted in the left $5^{\text {th }}$ intercostal space to drain the left massive hemothorax, draining about $1800 \mathrm{ml}$ (Figure 4). The next day, the patient required continuous veno-venous hemodialysis (CVVHD) for 24 hours due to renal impairment. Following the recovery of renal function, CVVHD was stopped. The patient also developed transient rapid atrial fibrillation, which converted using Amiodarone. The patient was extubated after 48 hours without any neurological deficits, was fully conscious and was able to move all four limbs. He complained of blurred vision and so a CT scan of the brain was performed, which exhibited a small infarction, which resolved after medical therapy. CT angiography of the thoracic aorta was repeated before discharge, which showed a good flow to the true lumen with thrombosis of the false lumen. The patient was discharged after full recovery and was prescribed antihypertension medications. One month later, CT angiography was repeated, which showed a good flow without endoleak or other problems with the stents. The patient was advised to continue his medications for hypertension and undergo a follow-up CT after six months.

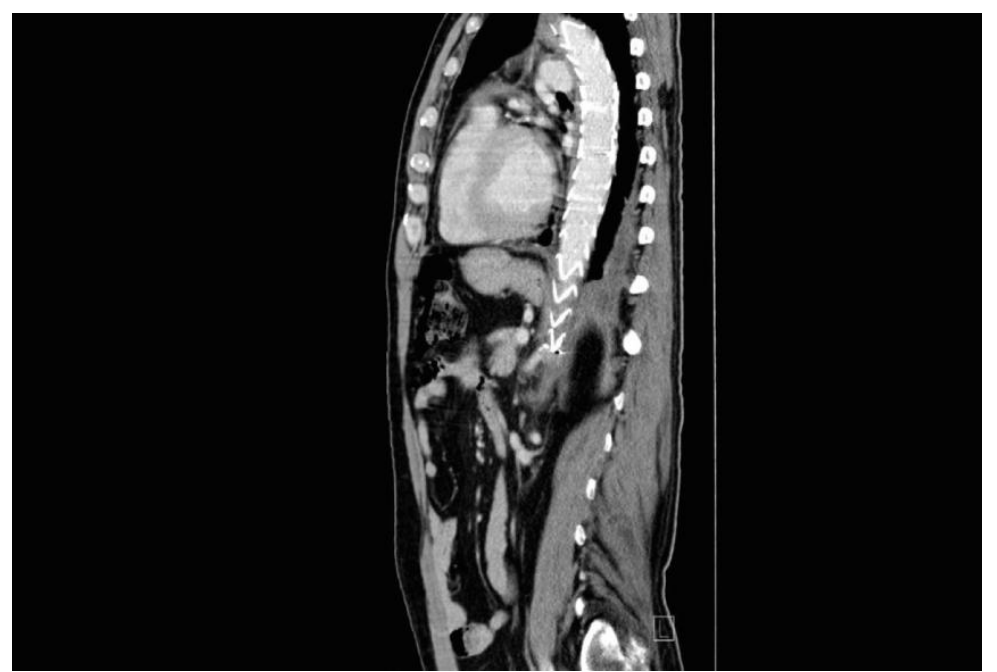

Figure 3:- Postoperative spiral CT scan indicating the location of the stents and an active descending thoracic active with good flow.

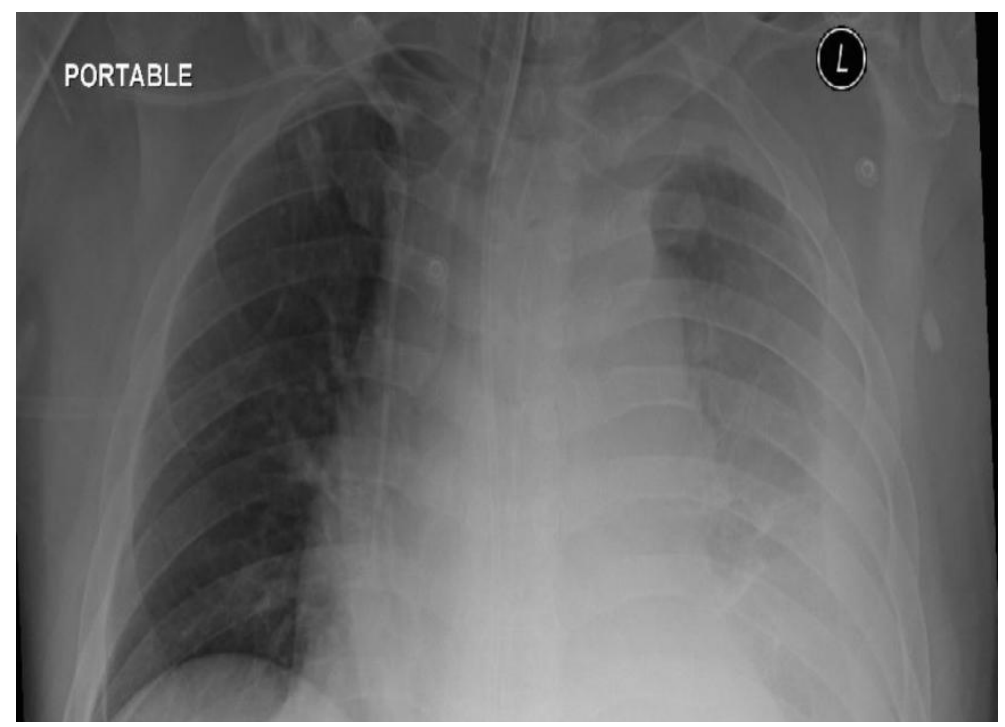

Figure 4:- Preoperative CXR, which showed a wide mediastinum and a large left hemothorax.

\section{Discussion:-}

Hemothorax caused by aortic dissection is an uncommon manifestation and results in a high mortality rate if left undiagnosed or untreated(McCloy et al., 1965).In this case, an elderly man presented with a massive hemothorax that was caused by type B thoracic aortic dissection. A classic aortic dissection is described by the presence of an 
intimal flap that separates the true and false lumens (Larson et al., 1984). There are two commonly accepted classification systems for dissections: Stanford and DeBakey. While the DeBakey classification was first proposed in 1965 (DeBakey et al., 1965) the Stanford classification system has become well established, particularly outside the cardiothoracic surgical community. This classification system describes only two types of AD: type A, which signifies the involvement of the ascending aorta, and type B, in which the ascending aorta is not affected (Daily et al., 1970). The most commonly presenting symptom is pain, which occurs in over $90 \%$ of patients, of which $85 \%$ report a sudden onset (Cigarroa et al., 1993). Typically, the pain is severe and sharp, which prompts patients to seek medical attention within minutes to hours of onset. It is also often reported to be categorically unlike any other pain they have experienced before. While pain location is typically assigned to the anterior chest in ascending (typeA) dissections, the pain is more often experienced in the back for descending (typeB) (Cigarroa et al., 1993). Chest pain is significantly more frequent in patients with type A dissection (83\% in type A dissection versus $71 \%$ in type B dissections) (Pape et al., 2015). Mean while, both back pain (64\% in type A dissection versus $47 \%$ in type B dissections) and abdominal pain (43\% in type A dissections versus $22 \%$ in type B dissections) are significantly more common with type B dissections (Cigarroa et al., 1993).

Several imaging modalities exist for diagnosing aortic dissections, including chest X-ray, TEE, CT, magnetic resonance imaging, conventional angiography, and a ortography. Each of these diagnostic modalities has some advantages and limitations (Willens et al, 1999). Chest x-rays normally show a widened mediastinum. In one study,69\% of patients with an aortic dissection were reported to have a widened mediastinum(Erbel et al., 1989). CT angiography is used initiallyin patients with assumed aortic dissection, especially in the emergency department setting where other studies are limited in availability. The majority of patients with suspected acute aortic dissection should be evaluated with both chest and abdominal dynamic contrast-enhanced, fine-cut CT scanning. Most importantly, three- dimensional CT scan reconstructions can aid treatment planning, and axial imaging improves the detection of topographic relationships of the true and false lumens and potential aortic branch compromise. CT is the most frequent first imaging modality performed due to its high sensitivity and specificity (Erbel et al., 1989; Nienaber et al., 1999). MRI has the highest accuracy and sensitivity for detection of all types of dissections, with the exception of DeBakey Type III aortic dissections, which can only be diagnosed with aortography. TEE is a widely used imaging technique for assessing unstable patients with suspected aortic pathologies since it can be performed quickly and simply at thepatient's bedside. Furthermore, it does not require any contrast material. Furthermore, the range of specificity reported for TEE ranged between $97 \%$ and 100\% across various studies (Bousnina et al., 200 7). Aortography is the gold standard for the diagnosis of an aortic dissection, but has largely yielded to CT angiography as the initial modality for diagnosing aortic dissection (Langin et al., 1994).

Following diagnosis, treatment optionsare informed by the type of lesion. Dissections of the ascending aorta (Stanford type A) must be operated upon emergently, while surgical repair of descending aortic dissections (Stanford type B) occurs in cases of acute complications (Slisatkorn et al., 2004). Endovascular stent grafting has been employed as a less invasive alternative to surgery, primarily in patients with complicated type B dissections (Tsai et al., 2005).

Hemothorax is defined as pleural effusion with a hematocrit level that is $\geq 50 \%$ of that of blood (Bousnina et al., 2007). The majority of hemothorax cases result from penetrating trauma or is iatrogenic in nature, following complicated invasive techniques, such as central venous catheters, pleural biopsy or thoracentesis. Others causes have been reported, such as hemostasis disorders that occur during anticoagulation therapy, hemophilia and thrombocytopenia (Little et al., 1999). Hemothorax that is secondary to rupture of a great chest vessel is uncommon, while hemothorax of vascular origin is often due to a rupture of the descending thoracic aorta, initially in the mediastinal and left pleural space, due to the proximity of the pleural cavity (McCloy et al., 1965), as was the case in the patient presented herein.

\section{References:-}

1. Bousnina, S., Zendah, I., Marniche, K., Racil, H.,Kach-boura, S., Megdiche, M. L., Chabbou, A. (2007): Left hemothorax caused by aortic dissection. Revue de Pneumologie Clinique, 2: 119-122.

2. Castaner,E.,Andreu,M., Gallardo,X., Mata, J.M.,Cabezuelo,M.A.,Pallardo,Y. (2003): CTinnontraumaticacutethoracicaortic disease: typicalandatypicalfeaturesandcomplications.Radiographics,23:93-110.

3. Cigarroa, J.E., Isselbacher, E.M., DeSanctis, R.W., Eagle, K.A. (1993): Diagnostic imaging in the evaluation of suspected aortic dissection. Old standards and new directions. N Engl J Med.,328: 35-43.

4. Daily, P.O., Trueblood, H.W., Stinson, E.B., Wuerflein, R.D., Shumway, N.E. (1970): Management of acute 
aortic dissections. Ann ThoracSurg, 10(3): 237-47.

5. Dake,M.D.,Thompson,M.,vanSambeek,M.,etal. (2013):DISSECT: anewmnemonic - based approach to the categorization of a ortic dissection. Eur J Vasc Endovasc Surg,46:175.

6. DeBakey, M.E., Henly, W.S., Cooley, D.A., Morris, G.C. Jr, Crawford, E.S., Beall, A.C. Jr. (1965): Surgical management of dissecting aneurysms of the aorta. J ThoracCardiovascSurg, 49: 130-49.

7. Erbel, R., Engberding, R., Daniel, W., Roelandt, J., Visser, C., Rennollet, H. (1989): Echocardiography in diagnosis of aortic dissection. Lancet, 1: 457-61.

8. Hagan,P.G.,Nienaber,C.A.,Isselbacher,E.M.,etal. (2000):TheInternationalRegistryofAcuteAorticDissection(IRAD):new insightsintoanolddisease.JAMA,283: 897.

9. Langin, T., Kelkel, P.E., Villemot, D. (1994): Left Hemothorax, a Complication of a Dissecting Aneurysm of the Descending Thoracic Aorta. Revue Des Maladies Respiratoires, 11(1): 74-76.

10. Larson, E.W., Edwards, W.D. (1984): Risk factors for aortic dissection: A necropsy study of 161 cases. Am J Cardiol,53:849-55.

11. Little, S., Johnson, J., Moon, B.Y., Mehta, S.(1999): Painless Left Hemorrhagic Pleural Effusion.Chest, 116(5): 1478-1480.

12. McCloy, R.M., Spittell, J.A., Jr, McGoon, D.C. (1965): The prognosis in aortic dissection (dissecting aortic hematoma or aneurysm) Circulation, 31: 665-9.

13. Nienaber, C.A., Fattori, R., Lund, G., et al. (1999): Nonsurgical reconstruction of thoracic aortic dissection by stent-graft placement. N Engl J Med., 340: 1539-45.

14. Pape,L.A.,Awais,M.,Woznicki,E.M.,etal. (2015) : Presentation, Diagnosis, and outcomes of acutea ortic dissection : 17 - year trends from the International Registry of Acute Aortic Dissection. J Am Coll Cardiol, 66:350.

15. Slisatkorn, W., Laksanabunsong, P., Thongcharoen, P. (2004): Ruptured Right Aortic Arch Aneurysm. Asian Cardiovascular and Thoracic Annals, 12(4): 360-362.

16. Tsai, T.T., Nienaber, C.A., Eagle, K.A. (2005): Acute aortic syndromes. Circulation,112: 3802.

17. Willens,H.J., Kessler,K.M. (1999): Transesophageal echocardiographyinthediagnosisof dis- eases of the thoracicaorta:part1. Aortic dissection,aorticintramural hematoma, and penetrating atheroscleroticulcer of the aorta.Chest, 116:1772-1779. 